OPEN ACCESS

Edited by:

Sukvinder Kaur Bhamra, University of Kent, United Kingdom

Reviewed by: Zemede Asfaw,

Addis Ababa University, Ethiopia Anthony Booker University of Westminster, United Kingdom

*Correspondence: Mihael Cristin Ichim cichim@hotmail.com

Specialty section:

This article was submitted to Ethnopharmacology, a section of the journal

Frontiers in Pharmacology

Received: 30 September 2020

Accepted: 25 November 2020

Published: 11 January 2021

Citation:

Ichim MC and de Boer HJ (2020) A

Review of Authenticity and

Authentication of Commercial Ginseng

Herbal Medicines

and Food Supplements.

Front. Pharmacol. 11:612071.

doi: 10.3389/fphar.2020.612071

\section{A Review of Authenticity and Authentication of Commercial Ginseng Herbal Medicines and Food Supplements}

\author{
Mihael Cristin Ichim ${ }^{1 \star}$ and Hugo J. de Boer ${ }^{2}$ \\ 1"Stejarul" Research Centre for Biological Sciences, National Institute of Research and Development for Biological Sciences, \\ Piatra Neamt, Romania, ${ }^{2}$ Natural History Museum, University of Oslo, Oslo, Norway
}

Ginseng traditional medicines and food supplements are the globally top selling herbal products. Panax ginseng, Panax quinquefolius and Panax notoginseng are the main commercial ginseng species in herbal medicine. Prices of ginseng products vary widely based on the species, quality, and purity of the used ginseng, and this provides a strong driver for intentional adulteration. Our systematic literature search has reviewed the authenticity results of 507 ginseng-containing commercial herbal products sold in 12 countries scattered across six continents. The analysis of the botanical and chemical identity of all these products shows that $76 \%$ are authentic while $24 \%$ were reported as adulterated. The number of commercial products as well as the percentage of adulteration varies significantly between continents, being highest in South America (100\%) and Australia (75\%), and lower in Europe (35\%), North America (23\%), Asia (21\%) and Africa (0\%). At a national level, from the five countries for which more than 10 products have been successfully authenticated, the highest percentage of adulterated ginseng products were purchased from Taiwan (49\%), followed by Italy (37\%), China $(21 \%)$, and USA (12\%), while all products bought in South Korea were reported to be authentic. In most cases, labeled Panax species were substituted with other Panax species, but substitution of ginseng root, the medicinally recommended plant part, with leaves, stems or flowers was also reported. Efficient and practical authentication using biomarkers to distinguish the main ginseng varieties and secondary metabolite spectra for age determination are essential to combat adulteration in the global marketplace.

Keywords: ginseng, Panax, identification, adulteration, contamination, herbal product, herbal medicine, food supplement

\section{INTRODUCTION}

Ginseng is used collectively to refer to several plant species, mainly in the Araliaceae genera Panax L. and Eleutherococcus Maxim. In China, the ginseng preparations have been used for thousands of years in traditional medicine (Robbins, 1998). Demand for ginseng roots in the 18th century intensified the wild harvest of the main species Panax ginseng C. A. Mey. (Araliaceae) (Korean ginseng), and nearly extirpated it from the wild (Millspaugh, 1974), but also fuelled a rapid expansion in wild-harvesting of Panax quinquefolius L. (Araliaceae) (American ginseng) that in turn destroyed 
wild populations in North America (Kimmens, 1975). There are currently 13 recognized ginseng species, but new taxa at species, subspecies and variety level are continuously published (Manzanilla et al., 2018). The most commonly used species in the genus are $P$. ginseng, $P$. quinquefolius, Panax notoginseng (Burkill) F. H. Chen (Araliaceae) (Chinese ginseng), Panax japonicus (T. Nees) C. A. Mey. (Araliaceae) (Japanese ginseng), Panax pseudoginseng Wall. (Araliaceae) (Himalayan ginseng) and Panax vietnamensis $\mathrm{Ha}$ and Grushv. (Araliaceae) (Vietnamese ginseng) (Yang and $\mathrm{Wu}, 2016$ ). The majority of commercialized ginseng material is from cultivation and controlled sustainable wild harvest, whereas material from uncontrolled depletive wild harvesting plays a minor and decreasing role. $P$. ginseng, $P$. quinquefolius and $P$. notoginseng are the three most widely used species in herbal medicine, and are traded as either red ginseng (Ginseng Radix et Rhizoma Rubra), white ginseng (Ginseng Radix et Rhizoma), American ginseng (Panacis Quinquefolii Radix), or notoginseng (Notoginseng Radix et Rhizoma). These ginseng varieties are sold in different stages of processing from raw materials through highly processed products that have lost their botanical morphological characteristics to extracts differentiated mainly by ginsenoside content (Yang and $\mathrm{Wu}, 2016$ ). In the global market, many different formulations of these herbs are available, including gelcaps, capsules, teas, tinctures, slices to eat in salads, powders, as well as entire roots. There are a wide variety of products that contain ginseng such as toothpaste, cigarettes, soaps, cosmetics, beverages (including beer), coffee, baby food, candies, and gum (Morgan and Cupp, 2010).

In the global medicinal plant trade, the economic value of ginseng is estimated to be more than US\$ 2.1 billion (Manzanilla et al., 2018). Prices vary widely based on the quality and the quantity of the ginseng root. The ginseng cultivar, locality, growth condition (cultivated or harvested from the wild), and plant age are some of the most important factors which influence the quality and properties of $P$. ginseng (Kim et al., 2012). It was shown previously that $P$. ginseng possesses different active ingredients and differentiated curative effects depending on the age of the plants, that is why the older ginseng plants are sold at a much higher price than the younger ones (Kim et al., 2011; Yang et al., 2012; Pan et al., 2013). Tenfold and more price differences among the main Panax processed varieties is the main driver for intentional adulteration (Choi et al., 2007). Adulteration using low-price varieties, low grade material from above-ground parts or processing waste, alternative species, or nothing at all, could bring huge profits through deceptive or illegal trade (Huang et al., 2017). The authentic herbal medicines and food supplements should be non-adulterated (Simmler et al., 2018) while the inadvertent or intentional adulteration (Simmler et al., 2018) includes contamination, product substitution and the use of fillers (Shanmughanandhan et al., 2016).

Efficient discrimination methods for ginseng varieties is necessary to combat fraud. Product adulteration and substitution are severe and widespread problems in the ginseng market, given the significant difference in medicinal value and economic benefits of different ginseng preparations (Zhao et al., 2020). The adulteration interferes with the proper and correct use of ginseng products and compromises the credibility of the whole supply chain. The development of efficient and practical authentication using biomarkers to distinguish the main ginseng varieties and secondary metabolite spectra for age determination are essential to combat adulteration. This review aims to provide an overview of the state of the art in ginseng authentication, while also highlighting current strengths and limitations.

\section{METHODS}

\section{Search Strategy}

Four databases (Web of Science, PubMed, Scopus, and ScienceDirect) were systematically searched for peer reviewed articles using keywords combined with Boolean operators: [(ginseng OR Panax) AND (identification OR authentication OR authenticity OR authentic OR adulteration OR contamination OR substitution)] following the PRISMA guidelines (Moher et al., 2009). After the search was performed (on 17 February 2020), weekly updates were received and taken into consideration as the option "search alert" has been activated for all four literature databases.

\section{Selection Process and Criteria}

A total of 3,683 records were identified, with $\mathrm{WoS}=1,277$, PubMed $=692$, Scopus $=1,480$, ScienceDirect $=234$ respectively, as well as another 49 records from other sources. Out of these a total 1,023 records were retained after removing duplicates. After screening, 869 records were excluded. The highly diverse reporting formats of the authenticity results made it necessary to define unifying criteria for selection and retain only the relevant articles in our review. The remaining 154 full-text articles were assessed for eligibility using the assessment criteria: 1) The reported samples had to be "herbal products" in the broad sense. The widest possible range of commercial names was used for the searches and accepted for inclusion in the review; 2) The analyzed products had to be "commercial." The "cost-free," "gifts" or the "donated" products were excluded from our analysis; 3) The products had to be allocated specifically to a "country" or "territory" (i.e., Hong Kong, European Union); 4) The "authentic" or "adulterated" conclusion was drawn by the authors of the reviewed articles; 5) All authentication methods were accepted. After applying these selection criteria, 120 articles were excluded because they did not report authentication results of commercial herbal products. This objective literature search identified 34 peer-reviewed publications reporting authentication of a total of 507 ginseng-containing commercial herbal products (Table 1). Authentication of botanical identity was reported for 468 products in 29 studies, and 13 of them used only DNAbased methods, mostly DNA barcoding. In another 13 studies only chemical methods were used, while the remainder used a combination of genetic and chemical authentication methods. Fifty-seven percent of studies (17 out of 30) reported authentication results for ginseng products purchased from China. The integrity of the chemical composition of 39 ginseng products was reported in five additional peerreviewed studies. 
TABLE 1 | The authenticity of ginseng commercial herbal products sold on the global market.

\begin{tabular}{|c|c|c|c|c|c|c|c|c|}
\hline \multirow{3}{*}{$\begin{array}{l}\text { Country/ } \\
\text { territory }\end{array}$} & \multirow{3}{*}{$\begin{array}{c}\text { Labeled and } \\
\text { authenticated } \\
\text { Panax } \\
\text { sp./type of } \\
\text { herbal product }\end{array}$} & \multirow{3}{*}{$\begin{array}{l}\text { Identified } \\
\text { adulterant }\end{array}$} & \multirow{3}{*}{$\begin{array}{c}\text { Product } \\
\text { composition }\end{array}$} & \multicolumn{3}{|c|}{ Products } & \multirow{3}{*}{$\begin{array}{l}\text { Authentication } \\
\text { methods }\end{array}$} & \multirow[t]{3}{*}{ Ref } \\
\hline & & & & Total & Authentic & Adulterated & & \\
\hline & & & & no & no & no & & \\
\hline \multicolumn{9}{|c|}{ Botanical identity } \\
\hline Brazil & P. ginseng & Pfaffia spp. & $\begin{array}{l}\text { Root products }(0.2 \% \\
\text { ginsenoside } \mathrm{Rg} 1 \text { and } \\
0.1 \% \text { ginsenoside } \\
\text { Rb1, HPLC) }\end{array}$ & 5 & 0 & 5 & DNA barcoding & $\begin{array}{l}\text { (Palhares et al., } \\
\text { 2015) }\end{array}$ \\
\hline China & $\begin{array}{l}P . \text { ginseng, } P \text {. } \\
\text { quinquefolius, } P \text {. } \\
\text { notoginseng }\end{array}$ & $\mathrm{n} / \mathrm{a}$ & $\begin{array}{l}\text { Batches of TCM } \\
\text { compound } \\
\text { preparations (e.g. pill, } \\
\text { bag, injection, } \\
\text { capsule, tablet, } \\
\text { powder, dripping pill) }\end{array}$ & 40 & 38 & 2 & LC-MS & (Yang et al., 2016) \\
\hline China & P. ginseng & P. quinquefolius & $\begin{array}{l}\text { Batches of CPMs } \\
\text { containing ginseng } \\
\text { products }\end{array}$ & 24 & 19 & 5 & DNA barcoding & (Liu et al., 2016) \\
\hline China & P. ginseng & P. quinquefolius & $\begin{array}{l}\text { Ginseng Radix et } \\
\text { Rhizoma samples }\end{array}$ & 15 & 12 & 3 & DNA barcoding & (Han et al., 2016) \\
\hline China & P. notoginseng & $\mathrm{n} / \mathrm{a}$ & $\begin{array}{l}\text { Baches of CPM } \\
\text { Shuxiong tablets } \\
\text { prepared from } \\
\text { Notoginseng Radix } \\
\text { et Rhizoma), } \\
\text { Carthami Flos, and } \\
\text { Chuanxiong } \\
\text { Rhizoma }\end{array}$ & 12 & 12 & 0 & $\begin{array}{l}\text { UPLC/QTOF- } \\
\text { Fast DDA }\end{array}$ & (Yao et al., 2016) \\
\hline China & $\begin{array}{l}P . \text { ginseng, } P \text {. } \\
\text { quinquefolius }\end{array}$ & $\mathrm{n} / \mathrm{a}$ & $\begin{array}{l}\text { CPMs containing } \\
\text { ginseng or American } \\
\text { ginseng }\end{array}$ & 11 & 8 & 3 & MAS-PCR & (Cheng et al., 2015) \\
\hline China & $\begin{array}{l}P . \text { ginseng, } P \text {. } \\
\text { quinquefolius, } P \text {. } \\
\text { notoginseng }\end{array}$ & $\mathrm{n} / \mathrm{a}$ & $\begin{array}{l}\text { TCHMs multi- } \\
\text { ingredient ginseng } \\
\text { preparations, Sheng } \\
\text { Mai Yin (SMY) } \\
\text { capsule and granules }\end{array}$ & 11 & 6 & 5 & HPTLC & (Xie et al., 2006) \\
\hline China & P. notoginseng & flower of $P$. notoginseng & $\begin{array}{l}\text { TCM preparations } \\
\text { with P. notoginseng } \\
\text { powder (dry roots } \\
\text { and rootstock) }\end{array}$ & 10 & 9 & 1 & UPLC/Qtof MS & (Liu et al., 2015) \\
\hline China & P. ginseng & $\mathrm{n} / \mathrm{a}$ & $\begin{array}{l}\text { Ginseng containing } \\
\text { samples }\end{array}$ & 10 & 8 & 2 & PCR & (Zhou et al., 2016) \\
\hline China & P. ginseng & $\begin{array}{l}\text { P. quinquefolius, } \\
\text { Platycodon grandiflorum } \\
\text { (Jacq.) A.DC. } \\
\text { (Campanulaceae), } \\
\text { Physochlaina } \\
\text { infundibularisKuang } \\
\text { (Solanaceae), Phytolacca } \\
\text { acinosa Roxb. } \\
\text { (Phytolaccaceae) }\end{array}$ & Root samples & 8 & 5 & 3 & NIR barcode & (Dong et al., 2020) \\
\hline China & P. ginseng & $\mathrm{n} / \mathrm{a}$ & $\begin{array}{l}\text { Ginseng Radix et } \\
\text { Rhizoma (dried roots } \\
\text { and rhizomes of } P \text {. } \\
\text { ginseng) }\end{array}$ & 3 & 3 & 0 & DNA barcoding & (Zhang et al., 2019) \\
\hline China & $\begin{array}{l}P . \text { ginseng, } P \text {. } \\
\text { notoginseng }\end{array}$ & $\mathrm{n} / \mathrm{a}$ & $\begin{array}{l}\text { steamed roots of } P \text {. } \\
\text { ginseng; powdered } \\
\text { roots of } P \text {. } \\
\text { notoginseng }\end{array}$ & 2 & 2 & 0 & DNA barcoding & (Dong et al., 2014) \\
\hline China & P. ginseng & $\mathrm{n} / \mathrm{a}$ & $\begin{array}{l}\text { Shihu Yeguang Pills } \\
\text { containing Ginseng } \\
\text { Radix Et Rhizoma }\end{array}$ & 1 & 1 & 0 & DNA barcoding & (Jiang et al., 2019) \\
\hline
\end{tabular}

(Continued) 
TABLE 1 | (Continued) The authenticity of ginseng commercial herbal products sold on the global market.

\begin{tabular}{|c|c|c|c|c|c|c|c|c|}
\hline \multirow{3}{*}{$\begin{array}{l}\text { Country/ } \\
\text { territory }\end{array}$} & \multirow{3}{*}{$\begin{array}{c}\text { Labeled and } \\
\text { authenticated } \\
\text { Panax } \\
\text { sp./type of } \\
\text { herbal product }\end{array}$} & \multirow{3}{*}{$\begin{array}{l}\text { Identified } \\
\text { adulterant }\end{array}$} & \multirow{3}{*}{$\begin{array}{l}\text { Product } \\
\text { composition }\end{array}$} & \multicolumn{3}{|c|}{ Products } & \multirow{3}{*}{$\begin{array}{l}\text { Authentication } \\
\text { methods }\end{array}$} & \multirow[t]{3}{*}{ Ref } \\
\hline & & & & Total & Authentic & Adulterated & & \\
\hline & & & & no & no & no & & \\
\hline China & P. ginseng & $\mathrm{n} / \mathrm{a}$ & $\begin{array}{l}\text { Batches of Asian } \\
\text { ginseng }\end{array}$ & 11 & 11 & 0 & UPLC/Q-TOF-MS & (Li et al., 2010) \\
\hline China & P. quinquefolius & $\mathrm{n} / \mathrm{a}$ & $\begin{array}{l}\text { Batches of American } \\
\text { ginseng }\end{array}$ & 7 & 7 & 0 & & \\
\hline Canada & P. quinquefolius & $\mathrm{n} / \mathrm{a}$ & $\begin{array}{l}\text { Batches of American } \\
\text { ginseng }\end{array}$ & 5 & 5 & 0 & & \\
\hline United States & P. quinquefolius & $\mathrm{n} / \mathrm{a}$ & $\begin{array}{l}\text { Batches of American } \\
\text { ginseng }\end{array}$ & 4 & 4 & 0 & & \\
\hline China & P. quinquefolius & P. ginseng & $\begin{array}{l}\text { Batches of American } \\
\text { ginseng preparations }\end{array}$ & 13 & 0 & 13 & & \\
\hline China & P. ginseng & leaf/stem & Root extract & 1 & 0 & 1 & HPTLC, HPLC & (Govindaraghavan, \\
\hline European Union & P. ginseng & $\begin{array}{l}P \text {. ginseng leaf or other } \\
\text { plant parts, } P \text {. quinquefolius } \\
\text { roots }\end{array}$ & $\begin{array}{l}\text { Root extracts, stem/ } \\
\text { leaf extract, berry } \\
\text { extract }\end{array}$ & 12 & 6 & 6 & & 2017) \\
\hline Australia & P. ginseng & $\begin{array}{l}P . \text { ginseng leaf or other } \\
\text { plant parts }\end{array}$ & $\begin{array}{l}\text { Capsules (extract or } \\
\text { herb), tablet }\end{array}$ & 4 & 1 & 3 & & \\
\hline South Korea & P. ginseng & $\mathrm{n} / \mathrm{a}$ & $\begin{array}{l}\text { P. ginseng extract, } \\
\text { tea, capsule }\end{array}$ & 3 & 3 & 0 & Multiplex PCR & (Tian et al., 2020) \\
\hline China & $\begin{array}{l}P . \text { ginseng, } P \text {. } \\
\text { quinquefolius }\end{array}$ & $\mathrm{n} / \mathrm{a}$ & $\begin{array}{l}P \text {. ginseng extract, } \\
\text { tea, capsule; } P \text {. } \\
\text { quinquefolius } \\
\text { extract, tea, capsule }\end{array}$ & 6 & 6 & 0 & & \\
\hline United States & $\begin{array}{l}P . \text { ginseng, } P \text {. } \\
\text { quinquefolius }\end{array}$ & $\mathrm{n} / \mathrm{a}$ & $\begin{array}{l}\text { P. quinquefolius } \\
\text { extract, tea, capsule }\end{array}$ & 3 & 3 & 0 & & \\
\hline Hong Kong & P. ginseng & $\mathrm{n} / \mathrm{a}$ & $\begin{array}{l}\text { Ready-to-serve } \\
\text { ginseng soup }\end{array}$ & 1 & 1 & 0 & $\begin{array}{l}\text { Multiplex PCR, } \\
\text { DNA sequencing }\end{array}$ & (Lo et al., 2015) \\
\hline Hong Kong & $\begin{array}{l}P . \text { ginseng, } P \text {. } \\
\text { quinquefolius }\end{array}$ & $\mathrm{n} / \mathrm{a}$ & $\begin{array}{l}\text { Dried roots, } \\
\text { powders, tea } \\
\text { granules }\end{array}$ & 7 & 7 & 0 & PCR, HAD & (Jiang et al., 2014) \\
\hline Italy & $\begin{array}{l}P . \text { ginseng, } P \text {. } \\
\text { quinquefolius }\end{array}$ & $\mathrm{n} / \mathrm{a}$ & $\begin{array}{l}\text { Raw materials (as } \\
\text { body root, root tails } \\
\text { and root prongs), } \\
\text { capsules and tablets } \\
\text { (containing dried } \\
\text { extract) }\end{array}$ & 19 & 12 & 7 & RFLP, HPLC & $\begin{array}{l}\text { (Del Serrone et al., } \\
\text { 2006) }\end{array}$ \\
\hline South Korea & P. ginseng & $\mathrm{n} / \mathrm{a}$ & $\begin{array}{l}\text { decoctions, } \\
\text { beverages, capsules, } \\
\text { tablets }\end{array}$ & 61 & 61 & 0 & $\begin{array}{l}\text { HPLC, } \\
\text { UPLC-DAD-ESI- } \\
\text { IT-TOF-MS }\end{array}$ & (Choi et al., 2018) \\
\hline South Korea & P. ginseng & $\mathrm{n} / \mathrm{a}$ & $\begin{array}{l}\text { Bak-Ho-Ga-Insam- } \\
\text { Tang resources } \\
\text { containing } P \text {. } \\
\text { ginseng }\end{array}$ & 14 & 14 & 0 & Gradient PCR & (Shim et al., 2005) \\
\hline Taiwan & P. ginseng & $\begin{array}{l}\text { not composed of } 6 \text { years } \\
\text { old ginseng radix only }\end{array}$ & $\begin{array}{l}\text { White ginseng radix } \\
\text { sliced material, } \\
\text { powder, capsules }\end{array}$ & 7 & 6 & 1 & $1 \mathrm{H}-\mathrm{NMR}$ & (Lin et al., 2010) \\
\hline China & P. ginseng & $\mathrm{n} / \mathrm{a}$ & $\begin{array}{l}\text { white ginseng radix } \\
\text { sliced material }\end{array}$ & 1 & 1 & 0 & & \\
\hline Taiwan & P. ginseng & Panacis quinquefolii Radix & $\begin{array}{l}\text { Chinese medical } \\
\text { preparations } \\
\text { containing Ginseng } \\
\text { Radix }\end{array}$ & 58 & 27 & 31 & $\begin{array}{l}\text { nested PCR, } \\
\text { RFLP, DNA } \\
\text { sequencing }\end{array}$ & (Lu et al., 2010) \\
\hline United Kingdom & $\begin{array}{l}P . \text { ginseng, } P \text {. } \\
\text { quinquefolius, } P \text {. } \\
\text { notoginseng }\end{array}$ & $\mathrm{n} / \mathrm{a}$ & $\begin{array}{l}\text { American ginseng, } \\
\text { white Asian ginseng, } \\
\text { sanchi ginseng }\end{array}$ & 8 & 8 & 0 & LC-MS & (Kite et al., 2003) \\
\hline United States & P. ginseng & $\begin{array}{l}\text { Astragalus propinquus } \\
\text { Schischkin (Leguminosae) }\end{array}$ & $\begin{array}{l}\text { Single } \\
\text { ingredient } \mathrm{HMP}\end{array}$ & 1 & 0 & 1 & DNA barcoding & (Molina et al., 2018) \\
\hline
\end{tabular}


TABLE 1 | (Continued) The authenticity of ginseng commercial herbal products sold on the global market.

\begin{tabular}{|c|c|c|c|c|c|c|c|c|}
\hline \multirow{2}{*}{$\begin{array}{l}\text { Country/ } \\
\text { territory }\end{array}$} & \multirow{2}{*}{$\begin{array}{c}\text { Labeled and } \\
\text { authenticated } \\
\text { Panax } \\
\text { sp./type of } \\
\text { herbal product }\end{array}$} & \multirow{2}{*}{$\begin{array}{l}\text { Identified } \\
\text { adulterant }\end{array}$} & \multirow{2}{*}{$\begin{array}{c}\text { Product } \\
\text { composition }\end{array}$} & \multicolumn{3}{|c|}{ Products } & \multirow{2}{*}{$\begin{array}{l}\text { Authentication } \\
\text { methods }\end{array}$} & \multirow[t]{2}{*}{ Ref } \\
\hline & & & & $\frac{\text { Total }}{\text { no }}$ & $\frac{\text { Authentic }}{\text { no }}$ & $\frac{\text { Adulterated }}{\text { no }}$ & & \\
\hline United States & $\begin{array}{l}P . \text { ginseng, } P \text {. } \\
\text { quinquefolius }\end{array}$ & $\mathrm{n} / \mathrm{a}$ & $\begin{array}{l}\text { Ground } P \text {. } \\
\text { quinquefolius root } \\
\text { (capsule), red } P \text {. } \\
\text { ginseng root extract } \\
\text { (liquid vial) }\end{array}$ & 2 & 2 & 0 & UPLC/QTOF-MS & (Yuk et al., 2016) \\
\hline United States & P. quinquefolius & P. ginseng & $\begin{array}{l}\text { American ginseng } \\
\text { products }\end{array}$ & 6 & 4 & 2 & HPLC & (Yu et al., 2014) \\
\hline United States & $\begin{array}{l}P . \text { ginseng, } P \text {. } \\
\text { quinquefolius }\end{array}$ & soybean & $\begin{array}{l}\text { American and } \\
\text { Korean ginseng fresh } \\
\text { or dried roots, } \\
\text { powders, capsules, } \\
\text { tablets }\end{array}$ & 24 & 22 & 2 & $\begin{array}{l}\text { PCR, RAPD, } \\
\text { HPLC }\end{array}$ & $\begin{array}{l}\text { (Mihalov et al., } \\
\text { 2000) }\end{array}$ \\
\hline China & $\begin{array}{l}P . \text { ginseng, } P \text {. } \\
\text { quinquefolius }\end{array}$ & $\mathrm{n} / \mathrm{a}$ & $\begin{array}{l}\text { American and } \\
\text { Korean ginseng dried } \\
\text { root }\end{array}$ & 2 & 2 & 0 & & \\
\hline $\begin{array}{l}\text { United States } \\
\text { and Canada }\end{array}$ & $\begin{array}{l}P . \text { ginseng, } P \text {. } \\
\text { quinquefolius }\end{array}$ & P. quinquefolius & $\begin{array}{l}\text { NHPs containing } \\
\text { Red Korean, Korean, } \\
\text { American, sand } \\
\text { ginseng (capsules, } \\
\text { tablets, roots, carved } \\
\text { roots, extracts, teas, } \\
\text { and dried and } \\
\text { shredded products }\end{array}$ & 36 & 22 & 14 & DNA barcoding & $\begin{array}{l}\text { (Wallace et al., } \\
\text { 2012) }\end{array}$ \\
\hline Total & & & & 468 & 358 & 110 & & \\
\hline & Chemic & al composition & & & & & & \\
\hline China & $\begin{array}{l}\text { Functional food for } \\
\text { relieving physical } \\
\text { fatigue }\end{array}$ & $\begin{array}{l}\text { Testosterone, adalafil, } \\
\text { sildenafil }\end{array}$ & $\begin{array}{l}\text { Fur seal ginseng pills } \\
\text { with complex herbal } \\
\text { composition, } \\
\text { including } P \text {. ginseng }\end{array}$ & 16 & 13 & 3 & HPLC & (Wang et al., 2019) \\
\hline China & $\begin{array}{l}\text { Antidiabetic } \\
\text { functional food }\end{array}$ & $\begin{array}{l}\text { Tolbutamide, glimepiride, } \\
\text { metformin }\end{array}$ & $\begin{array}{l}\text { Bitter melon and } \\
\text { ginseng soft gels } \\
\text { containing also } \\
\text { containing American } \\
\text { ginseng root }\end{array}$ & 16 & 13 & 3 & $\begin{array}{l}\text { UPLC-Q-Orbitrap- } \\
\text { MS/MS }\end{array}$ & (Xie et al., 2019) \\
\hline China & $\begin{array}{l}\text { Herbal medicines for } \\
\text { male sexual health }\end{array}$ & $\begin{array}{l}\text { Sildenafil, hongdenafil, } \\
\text { vardenafil, homosildenafil }\end{array}$ & $\begin{array}{l}\text { Complex herbal } \\
\text { composition, } \\
\text { including Radix et } \\
\text { rhizoma ginseng or } \\
\text { Radix et rhizoma } \\
\text { ginseng rubra (tablet, } \\
\text { capsule, pills, soft gel } \\
\text { capsule) }\end{array}$ & 4 & 0 & 4 & TLC, HPLC-MS & (Cai et al., 2010) \\
\hline Saudi Arabia & $\begin{array}{l}\text { Dietary supplements } \\
\text { for weight loss, } \\
\text { slimming and as a } \\
\text { stimulant or stamina } \\
\text { enhancer }\end{array}$ & $\begin{array}{l}\text { Theobromine, theophylline, } \\
\text { pseudoephedrine, caffeine, } \\
\text { hydrochlorothiazide, } \\
\text { yohimbine }\end{array}$ & $\begin{array}{l}\text { Ginseng extract } \\
\text { (capsule), Korean } \\
\text { Ginseng (capsule) }\end{array}$ & 2 & 2 & 0 & UHPLC-DAD & (Ahmad et al., 2020) \\
\hline Sweden & Dietary supplement & Ephedrine & Ginseng preparation & 1 & 0 & 1 & $\mathrm{n} / \mathrm{a}$ & (Cui et al., 1994) \\
\hline Total & & & & 39 & 28 & 11 & & \\
\hline TOTAL & & & & 507 & 386 & 121 & & \\
\hline
\end{tabular}

\section{RESULTS}

The analysis of the botanical and chemical identity of 507 ginsengcontaining commercial herbal products shows that $76 \%(n=386)$ are authentic while $24 \%(n=121)$ were reported as adulterated. These ginseng commercial herbal products were purchased from 12 countries scattered across six continents: Asia $(n=375)$, North
America $(n=81)$, Europe $(n=40)$, South America $(n=5)$, Australia $(n=4)$, and Africa $(n=2)$. Among continents, the number of commercial products as well as the percentage of adulteration varies significantly, being the highest in South America $(100 \%, n=5)$ and Australia $(75 \%, n=3)$, and lower in Europe $(35 \%, n=14)$, North America $(23 \%, n=19)$, Asia $(21 \%$, $n=80)$ and Africa (0\%) (Table 1). 
TABLE 2 | National distribution of the ginseng commercial ginseng herbal products and their authenticity.

\begin{tabular}{lccccc}
\hline Country & Products (total) & \multicolumn{4}{c}{ Authentic/adulterated } \\
& no & no & $\%^{*}$ & no & $\%^{*}$ \\
China & 224 & 176 & $79 \%$ & 48 & $21 \%$ \\
South Korea & 78 & 78 & $100 \%$ & 0 & $0 \%$ \\
Taiwan & 65 & 33 & $51 \%$ & 32 & $49 \%$ \\
United States & 40 & 35 & $88 \%$ & 5 & $12 \%$ \\
Italy & 19 & 12 & $63 \%$ & 7 & $37 \%$ \\
Hong Kong & 8 & 8 & $100 \%$ & 0 & $0 \%$ \\
United Kingdom & 8 & 8 & $100 \%$ & 0 & $0 \%$ \\
Brazil & 5 & 0 & $0 \%$ & 5 & $100 \%$ \\
Canada & 5 & 5 & $100 \%$ & 0 & $0 \%$ \\
Australia & 4 & 1 & $25 \%$ & 3 & $75 \%$ \\
Saudi Arabia & 2 & 2 & $100 \%$ & 0 & $0 \%$ \\
Sweden & 1 & 0 & $0 \%$ & 1 & $100 \%$
\end{tabular}

${ }^{*}$ The percentage values were rounded to the nearest whole number.

Analysis of authentication results per country $(n=459$ commercial ginseng products were clearly allocated to a single country) show that the number of samples purchased and tested for each country varies. In China, 224 ginseng products were successfully authenticated, representing almost half (49\%) of all reported samples worldwide, while South Korea $(n=78)$, Taiwan $(n=65)$ and United States $(n=40)$ follow distantly. All of these countries, except Taiwan, are the three major ginseng cultivating countries in the world. This data also suggests the importance of these herbal products in Asian traditional medicine systems is mirrored by the interest of the scientific community to develop and test new methods on market products. Much smaller numbers of tested commercial ginseng products were reported for Italy $(n=$ $19)$, Hong Kong $(n=8)$, United Kingdom $(n=8)$, Brazil $(n=5)$, Canada $(n=5)$, Australia $(n=4)$, Saudi Arabia $(n=2)$, and Sweden $(n=1)$, but they support and reconfirm the widespread interest for ginseng-containing products on the global market.

The ginseng authenticity reported for the 12 countries represented in our review range widely from country to country. The five countries for which more than 10 products have been successfully authenticated account together for $84 \%$ of all ginseng products analyzed worldwide. Out of these countries, the highest percentage of adulterated ginseng products were purchased from Taiwan (49\%), followed by Italy (37\%), China (21\%), United States (12\%), while all the products bought from the South Korean market were reported to be authentic. Notably, six out of the seven remaining countries, each with less than 10 authenticated commercial samples, have all their products reported either as authentic (i.e., Canada, Hong Kong, Saudi Arabia, United Kingdom), or adulterated (i.e., Brazil, Sweden) while the ginseng products purchased from Australia were reported to be $25 \%$ authentic and $75 \%$ adulterated (Table 2 ).

\section{DISCUSSION}

\section{Authenticity of Commercial Ginseng Herbal Products Sold on the Global Market}

Overall, the peer-reviewed publications show that one in each four ginseng containing herbal products sold on the market is adulterated with respect to the labeled species or declared chemical composition. Adulterated ginseng products are present across many regions and countries, and this confirms a recent global analysis of DNA-based authenticity testing, both in terms of adulteration percentage and geographic spread (Ichim, 2019). In addition, our analysis further confirms the widespread presence of adulterated commercial herbal products in traditional medicine systems, including Traditional Chinese Medicine (TCM) (Han et al., 2016) and Ayurvedic medicine (Seethapathy et al., 2019).

In most cases, labeled Panax species are substituted with other Panax species. P. ginseng is replaced by P. quinquefolius in China (Han et al., 2016; Liu et al., 2016; Dong et al., 2020) and Taiwan (Lu et al., 2010), and vice-versa in United States (Li et al., 2010; Yu et al., 2014), so that the species most highly valued in that country is substituted by other ginseng species. The adulteration of traditional ginseng medicinal products included the substitution of ginseng root, the medicinally recommended plant part, with leaves, stems (Govindaraghavan, 2017) or even flowers (Liu et al., 2015), all replacing the high value herbal material with less costly ones. Liu et al. (2017) showed that ginsenoside ratios could be used to determine the age of cultivated Panax ginseng with 30-fold differences in some ginsenosides with age. Premium ginseng commercial products sold on the Taiwanese market have been reported to be adulterated with inferior plant material (Lin et al., 2010). Economically motivated adulteration of commercial ginseng products includes the use of soybean as the only plant ingredient identified in commercial products sold in the United States (Mihalov et al., 2000) thus confirming the reported widespread adulteration of commercial herbal products on the North American market (Newmaster et al., 2013).

The widespread use of the ginseng vernacular name across many countries and continents, including for species not from the same genus or even family (Osathanunkul and Madesis, 2019), is a catalyst conducive to adulteration. In Brazil for example, ginseng products have been found to be adulterated with a completely unrelated species from another family, Pfaffia spp., albeit locally known as Brazilian ginseng (Palhares et al., 2015). The inadvertent contamination through plant misidentification during harvesting or cross-contamination during processing but also the intentional and fraudulent use of filler species or cheaper substitutes, present on the herbal product market (Jordan et al., 2010; Sgamma et al., 2017; Ichim et al., 2020), also affects the highly valued ginseng-containing food supplements and traditional medicines across the globalized market. The presence of substitutes or filler species in some cases reflects intentional, economically motivated and fraudulent practices by producers or vendors although the European Pharmacopoeia (Ph. Eur.) and the United States Pharmacopeia (USP) as well as some monographs for herbal raw materials, allow a certain amount (e.g., 2\% in USP) of foreign organic matter (Parveen et al., 2016; Sgamma et al., 2017) as acceptable accidental contamination. Counterfeiting by adding synthetic prescription drugs as chemical adulterants is another form of falsification, and Calahan et al. (2016) found that $28 \%$ of authenticated ginseng 
products in their study were adulterated. Apart from the many cases of adulteration and general low quality of commercial herbal products, $P$. ginseng was also reported, along with some other plant species, as adulterant of a herbal product sold in Australia, supposedly to contain only Eucalyptus radiata A. Cunn. ex DC. (Myrtaceae) and Melaleuca alternifolia (Maiden and Betche) Cheel (Myrtaceae). This latter case suggests either accidental contamination through poor manufacturing process or intentional adulteration for achieving an expected physiological or pharmacological effect (Hoban et al., 2020).

\section{Ginseng-Drug Interactions, Abuse and Negative Effects of Long-Term Use on Human Health}

The presence of unlabeled species, plant extracts or synthetic chemical compounds might negatively interact with other medicinal plants, food supplements or prescription drugs and will pose significant risks for human health (Jordan et al., 2010). Adverse drug reactions (ADRs) due to herb-drug interactions (HDI) can appear in patients taking herbs and prescribed medications concomitantly (Awortwe et al., 2018). Ginseng can interfere with various drugs, such as digoxin, insulin, anticoagulants, and monoamine oxidase inhibitors (Sellami et al., 2018). Pharmacovigilance relies heavily on ADR reporting, and despite initiatives to stimulate reporting of suspected ADRs associated with herbal medicines, numbers of herbal ADR reports are relatively low. ADRs Underreporting is likely to be specific for herbal medicines, since their users usually do not look for medical advice about their use of such products, or report if they experience any adverse effects (Barnes, 2003).

The specific active constituents in Panax herbs, the ginsenosides, have been shown to improve immune function, reduce mental stress, and stabilize blood pressure while ginseng products are used as an endurance performance enhancer (Sellami et al., 2018). Nevertheless, authentic ginseng products does not represent a doping concern for athletes, as there were no positive tests for any International Olympic Committee (IOC) banned or restricted substances in any of the subjects after the ingestion of commercially available, proprietary ginseng root extract product (Goel et al., 2004).

Adverse drug reactions to ginseng are associated with high doses and long-term usage (Kiefer and Pantuso, 2003; Sellami et al., 2018). The ginseng abuse syndrome includes edema, decreased appetite, depression, and hypotension ( $10 \%)$, hypertension $(17 \%)$, sleeplessness $(20 \%)$, nervousness $(25 \%)$, skin eruption (25\%), and morning diarrhea (35\%) (Paik and Lee, 2015). Long term use may cause blood clotting (Mohammed Abdul et al., 2018). Ginseng reduces the blood levels of warfarin and alcohol as well as induced mania if taken concomitantly with phenelzine, a non-selective and irreversible monoamine oxidase inhibitor used as an antidepressant and anxiolytic agent (Chen et al., 2011). Because the ginsenosides have a chemical structure similar to that of testosterone, estrogen, and glucocorticoids, the ginseng may also produce effects similar to those of estrogen (Anadón et al., 2016). Moreover, women may experience additional side effects, such as vaginal bleeding and breast tenderness. Most of these side effects are serious enough to warrant stopping taking ginseng in breast cancer patients (Sellami et al., 2018). A rare adverse drug reaction to herbal and dietary supplementation, the drug-induced liver injury (DILI), was reported as result from ingestion of ginseng for premenopausal symptoms (Lin et al., 2018). Recently, a website with a critically reviewed database presenting reported cases of ginseng-drug interactions was publicly launched ( $\mathrm{Wu}$ et al., 2019).

The herbal medicines differ considerably from conventional medicines, and they pose a variety of challenges to their pharmacovigilance. For herbal pharmacovigilance, four main challenges: 1) Substitution and adulteration; 2) Nomenclature of herbals and ingredients of plant origin; 3) Lack of monitoring; and 4) Standardization, have been identified (de Boer et al., 2015). This review highlights that all four apply to ginseng commercial products, and support the challenge of detecting significant adverse drug reactions in a timely manner to protect consumers.

\section{CONCLUSION}

Despite being the most valuable herbal product in terms of market value share, ginseng products are poorly regulated. Several authentication studies have shown that adulteration is not uncommon and not limited to any specific country of origin. The high market value of ginseng provides an incentive for fraudulent actors to generate profits at the expense of gullible consumers and honest producers, wholesalers and retailers. Although authentication using traditional, pharmacopoeial, analytical methods such as TLC, HPLC and NMR can be used for advanced quality control, the standard authentication protocols are insufficient for efficiently detecting species adulteration, adulteration with synthetic pharmaceuticals and spiking of low quality products with marker compounds. Cutting-edge approaches enable distinction of age-specific metabolite spectra, quantification of active ingredients and accurate identification of ginseng species, but these are not yet in widespread use.

\section{AUTHOR CONTRIBUTIONS}

MI performed the literature systematic search and analyzed the results. MI and $\mathrm{HB}$ wrote the manuscript together.

\section{FUNDING}

This publication was supported by the National Core Program funded by the Romanian Ministry of Research and Innovation, project number 25N/11.02.2019, BIODIVERS 19270401 (for MI). 


\section{REFERENCES}

Ahmad, R., Ahmad, N., AlHudaithi, N., AlHebshi, A., and Bukhari, A. (2020). Extraction and UHPLC-DAD detection of undeclared substances in marketavailable dietary supplements and slimming products in Eastern region, Saudi Arabia: an application of principal component analysis. Biomed. Chromatogr. 34, e4698. doi:10.1002/bmc.4698

Anadón, A., Martínez-Larrañaga, M. R., Ares, I., and Martínez, M. A. (2016). Interactions between nutraceuticals/nutrients and therapeutic drugs. Nutraceuticals 60, 855-874. doi:10.1016/B978-0-12-802147-7.00060-7

Awortwe, C., Makiwane, M., Reuter, H., Muller, C., Louw, J., and Rosenkranz, B. (2018). Critical evaluation of causality assessment of herb-drug interactions in patients. Br. J. Clin. Pharmacol. 84, 679-693. doi:10.1111/bcp.13490

Barnes, J. (2003). Pharmacovigilance of herbal medicines: a United Kingdom perspective. Drug Saf. 26, 829-851. doi:10.2165/00002018-200326120-00001

Cai, Y., Cai, T.-G., Shi, Y., Cheng, X.-L., Ma, L.-Y., Ma, S.-C., et al. (2010). Simultaneous determination of eight PDE5-IS potentially adulterated in herbal dietary supplements with TLC and HPLC-PDA-MS methods. J. Liq. Chromatogr. Relat. Technol.33, 1287-1306. doi:10.1080/10826076.2010.488979

Calahan, J., Howard, D., Almalki, A. J., Gupta, M. P., and Calderón, A. I. (2016). Chemical adulterants in herbal medicinal products: a review. Planta Med. 82, 505-515. doi:10.1055/s-0042-103495

Chen, X. W., Serag, E. S., Sneed, K. B., Liang, J., Chew, H., Pan, S. Y., et al. (2011). Clinical herbal interactions with conventional drugs: from molecules to maladies. Curr. Med. Chem. 18, 4836-4850. doi:10.2174/092986711797535317

Cheng, C. S., Tan, T. Q., Long, Z., Liu, Z. Z., and Zhou, H. (2015). Optimization of DNA extraction for Chinese patent medicine and its application on molecular identification of ginseng preparations by MAS-PCR. Chin. Tradit. Herbal Drugs 46, 2549-2555. doi:10.7501/j.issn.0253-2670.2015.17.008

Choi, J. Y., Hong, J. H., Dang, Y. M., Jamila, N., Khan, N., Jo, C. H., et al. (2018). Identification markers of adulteration in Korean red ginseng (Panax ginseng) products using high-performance liquid chromatography (HPLC) and liquid chromatography-mass spectrometry (LC-MS). Anal. Lett. 51, 2588-2601. doi:10.1080/00032719.2018.1443340

Choi, Y. E., Kim, Y. S., Yi, M. J., Park, W. G., Yi, J. S., Chun, S. R., et al. (2007). Physiological and chemical characteristics of field-and mountain-cultivated ginseng roots. J. Plant Biol. 50, 198-205. doi:10.1007/BF03030630

Cui, J., Garle, M., Eneroth, P., and Björkhem, I. (1994). What do commercial ginseng preparations contain?. Lancet. 344, 134. doi:10.1016/s0140-6736(94) 91322-6

de Boer, H. J., Ichim, M. C., and Newmaster, S. G. (2015). DNA barcoding and pharmacovigilance of herbal medicines. Drug Saf. 38, 611-620. doi:10.1007/ s40264-015-0306-8

Del Serrone, P., Attorri, L., Gallinella, B., Gallo, F. R., Federici, E., and Palazzino, G. (2006). Molecular identification of Panax ginseng C. A. Meyer in ginseng commercial products. Nat. Prod. Commun. 1, 1934578X0600101. doi:10.1177/ $1934578 X 0600101213$

Dong, F., Lin, J., You, J., Ji, J., Xu, X., Zhang, L., et al. (2020). A chemometric modeling-free near infrared barcode strategy for smart authentication and geographical origin discrimination of Chinese ginseng. Spectrochim. Acta A Mol. Biomol. Spectrosc. 226, 117555. doi:10.1016/j.saa.2019.117555

Dong, W., Liu, H., Xu, C., Zuo, Y., Chen, Z., and Zhou, S. (2014). A chloroplast genomic strategy for designing taxon specific DNA mini-barcodes: a case study on ginsengs. BMC Genet. 15, 138. doi:10.1186/s12863-014-0138-z

Goel, D. P., Geiger, J. D., Shan, J. J., Kriellaars, D., and Pierce, G. N. (2004). Dopingcontrol urinalysis of a ginseng extract, Cold-FX, in athletes. Int. J. Sport Nutr. Exerc. Metab. 14, 473-480. doi:10.1123/ijsnem.14.4.473

Govindaraghavan, S. (2017). Multiple ginsenosides ratios pattern-a pointer to identify Panax ginseng root extracts adulterated with other plant parts? Fitoterapia. 121, 64-75. doi:10.1016/j.fitote.2017.06.024

Han, J., Pang, X., Liao, B., Yao, H., Song, J., and Chen, S. (2016). An authenticity survey of herbal medicines from markets in China using DNA barcoding. Sci. Rep. 6, 18723. doi:10.1038/srep 18723

Hoban, C. L., Musgrave, I. F., Byard, R. W., Nash, C., Farrington, R., Maker, G., et al. (2020). Combined liquid chromatography-mass spectrometry and nextgeneration DNA sequencing detection of adulterants and contaminants in analgesic and anti-inflammatory herbal medicines. Pharmaceut. Med. 34, 49-61. doi:10.1007/s40290-019-00314-y

Huang, B., Chen, T., Xiao, S., Zha, Q., Luo, P., Wang, Y., et al. (2017). A new approach for authentication of four ginseng herbs and their related products based on the simultaneous quantification of 19 ginseng saponins by UHPLCTOF/MS coupled with OPLS-DA. RSC Adv. 7, 46839-46851. doi:10.1039/ C7RA06812C

Ichim, M. C., Häser, A., and Nick, P. (2020). Microscopic authentication of commercial herbal products in the globalized market: potential and limitations. Front. Pharmacol. 11, 876. doi:10.3389/fphar.2020.00876

Ichim, M. C. (2019). The DNA-based authentication of commercial herbal products reveals their globally widespread adulteration. Front. Pharmacol. 10, 1227. doi:10.3389/fphar.2019.01227

Jiang, L.-L., Wong, K.-L., Wong, Y.-L., Chen, W.-T., Li, M., Lau, C. B.-S., et al. (2014). Helicase-dependent amplification is effective in distinguishing Asian ginseng from American ginseng. Food Control. 43, 199-205. doi:10.1016/j. foodcont.2014.03.016

Jiang, W., Ren, L., Guo, M., Mantri, N., Zhao, S., and Pang, X. (2019). Detecting schisandrae chinensis fructus and its Chinese patent medicines with a nucleotide signature. Genes. 10. doi:10.3390/genes10050397

Jordan, S. A., Cunningham, D. G., and Marles, R. J. (2010). Assessment of herbal medicinal products: challenges, and opportunities to increase the knowledge base for safety assessment. Toxicol. Appl. Pharmacol. 243, 198-216. doi:10. 1016/j.taap.2009.12.005

Kiefer, D., and Pantuso, T. (2003). Panax ginseng. Am. Fam. Physician. 68, 1539-1542.

Kim, N., Kim, K., Choi, B. Y., Lee, D., Shin, Y. S., Bang, K. H., et al. (2011). Metabolomic approach for age discrimination of Panax ginseng using UPLCQ-tof MS. J. Agric. Food Chem. 59, 10435-10441. doi:10.1021/jf201718r

Kim, N., Kim, K., Lee, D., Shin, Y. S., Bang, K. H., Cha, S. W., et al. (2012). Nontargeted metabolomics approach for age differentiation and structure interpretation of age-dependent key constituents in hairy roots of Panax ginseng. J. Nat. Prod. 75, 1777-1784. doi:10.1021/np300499p

Kimmens, A. C. (1975). Tales of the Ginseng. New York, NY: William Morrow and Company Inc.

Kite, G. C., Howes, M. J., Leon, C. J., and Simmonds, M. S. (2003). Liquid chromatography/mass spectrometry of malonyl-ginsenosides in the authentication of ginseng. Rapid Commun. Mass Spectrom. 17, 238-244. doi: $10.1002 / \mathrm{rcm} .899$

Li, L., Luo, G. A., Liang, Q. L., Hu, P., and Wang, Y. M. (2010). Rapid qualitative and quantitative analyses of Asian ginseng in adulterated American ginseng preparations by UPLC/Q-TOF-MS. J. Pharm. Biomed. Anal. 52, 66-72. doi:10. 1016/j.jpba.2009.12.017

Lin, K., Lin, A. N., Linn, S., Hlaing, P. P., Vasudevan, V., and Reddy, M. (2018). Ginseng-related drug-induced liver injury. Case Rep. Gastroenterol. 12, 439-446. doi:10.1159/000490525

Lin, W. N., Lu, H. Y., Lee, M. S., Yang, S. Y., Chen, H. J., Chang, Y. S., et al. (2010). Evaluation of the cultivation age of dried ginseng radix and its commercial products by using (1)H-NMR fingerprint analysis. Am. J. Chin. Med. 38, 205-218. doi:10.1142/S0192415X10007762

Liu, P., Yu, H. S., Zhang, L. J., Song, X. B., Kang, L. P., Liu, J. Y., et al. (2015). A rapid method for chemical fingerprint analysis of Pan Panax notoginseng powders by ultra performance liquid chromatography coupled with quadrupole time-offlight mass spectrometry. Chin. J. Nat. Med. 13, 471-480. doi:10.1016/S18755364(15)30042-X

Liu, Y., Wang, X., Wang, L., Chen, X., Pang, X., and Han, J. (2016). A nucleotide signature for the identification of American ginseng and its products. Front. Plant Sci. 7, 319. doi:10.3389/fpls.2016.00319

Liu, Z., Wang, C. Z., Zhu, X. Y., Wan, J. Y., Zhang, J., Li, W., et al. (2017). Dynamic changes in neutral and acidic ginsenosides with different cultivation ages and harvest seasons: identification of chemical characteristics for Panax ginseng quality control. Molecules 22, 734. doi:10.3390/molecules 22050734

Lo, Y. T., Li, M., and Shaw, P. C. (2015). Identification of constituent herbs in ginseng decoctions by DNA markers. Chin. Med. 10, 1. doi:10.1186/s13020015-0029-x

Lu, K.-T., Lee, H.-C., Liu, F.-S., Lo, C.-F., and Lin, J.-H. (2010). Identification of ginseng radix in Chinese medicine preparations by nested PCR-DNA 
sequencing method and nested PCR-restriction fragment length polymorphism. J. Food Drug Anal. 18, 58-63. doi:10.38212/2224-6614.2223

Manzanilla, V., Kool, A., Nguyen Nhat, L., Nong Van, H., Le Thi Thu, H., and de Boer, H. J. (2018). Phylogenomics and barcoding of Panax: toward the identification of ginseng species. BMC Evol. Biol. 18, 44. doi:10.1186/s12862018-1160-y

Mihalov, J. J., Marderosian, A. D., and Pierce, J. C. (2000). DNA identification of commercial ginseng samples. J. Agric. Food Chem. 48, 3744-3752. doi:10.1021/ if000011b

Millspaugh, C. F. (1974). American medicinal plants: an illustrated and descriptive guide to plants indigenous to and naturalized in the United States which are used in medicine. New York, NY: Missouri Botanical Garden.

Mohammed Abdul, M. I., Siddique, S., Rahman, S. A. U., Lateef, D., Dan, S., Mandal, P., et al. (2018). A critical insight of modern herbal drugs therapy under the purview of toxicity and authenticity. Biomed. Res. 29. doi:10.4066/ biomedicalresearch.29-18-968

Moher, D., Liberati, A., Tetzlaff, J., and Altman, D. G.PRISMA Group (2009). Preferred reporting items for systematic reviews and meta-analyses: the PRISMA statement. PLoS Med. 6, e1000097. doi:10.1371/journal.pmed.1000097

Molina, J., Sherpa, C., Ng, J., Sonam, T., and Stuhr, N. (2018). DNA barcoding of online herbal supplements: crowd-sourcing pharmacovigilance in high school. Open Life Sci. 13, 48-55. doi:10.1515/biol-2018-0007

Morgan, A., and Cupp, M. J. (2010). "Panax ginseng," in Toxicology and clinical Pharmacology of herbal products. Forensic science and medicine. Editor M. J. Cupp (Totowa, NJ: Humana Press), 325.

Newmaster, S. G., Grguric, M., Shanmughanandhan, D., Ramalingam, S., and Ragupathy, S. (2013). DNA barcoding detects contamination and substitution in North American herbal products. BMC Med. 11, 222. doi:10.1186/1741-7015-11-222

Osathanunkul, M., and Madesis, P. (2019). Bar-HRM: a reliable and fast method for species identification of ginseng (Panax ginseng, Panax notoginseng, Talinum paniculatum and Phytolacca Americana). PeerJ. 7, e7660. doi:10. 7717/peerj.7660

Paik, D. J., and Lee, C. H. (2015). Review of cases of patient risk associated with ginseng abuse and misuse. J. Ginseng Res. 39, 89-93. doi:10.1016/j.jgr.2014.11.005

Palhares, R. M., Gonçalves Drummond, M., B.Pereira Cosenza, G., das Graças Lins Brandão, M., and Oliveira, G. (2015). Medicinal plants recommended by the world health organization: DNA barcode identification associated with chemical analyses guarantees their quality. PLoS One. 10, e0127866. doi:10. 1371/journal.pone.0127866

Pan, H. Y., Qu, Y., Zhang, J. K., Kang, T. G., and Dou, D. Q. (2013). Antioxidant activity of ginseng cultivated under mountainous forest with different growing years. J. Ginseng Res. 37, 355-360. doi:10.5142/jgr.2013.37.355

Parveen, I., Gafner, S., Techen, N., Murch, S. J., and Khan, I. A. (2016). DNA barcoding for the identification of botanicals in herbal medicine and dietary supplements: strengths and limitations. Planta Med. 82, 1225-1235. doi:10. 1055/s-0042-111208

Robbins, C. S. (1998). American ginseng: the root of North America's medicinal herb trade. Washington, D.C.: TRAFFIC North America, 94.

Seethapathy, G. S., Raclariu-Manolica, A. C., Anmarkrud, J. A., Wangensteen, H., and de Boer, H. J. (2019). DNA metabarcoding authentication of ayurvedic herbal products on the european market raises concerns of quality and fidelity. Front. Plant Sci. 10, 68. doi:10.3389/fpls.2019.00068

Sellami, M., Slimeni, O., Pokrywka, A., Kuvačić, G., D Hayes, L., Milic, M., et al. (2018). Herbal medicine for sports: a review. J. Int. Soc. Sports Nutr. 15, 14. doi:10.1186/s12970-018-0218-y

Sgamma, T., Lockie-Williams, C., Kreuzer, M., Williams, S., Scheyhing, U., Koch, E., et al. (2017). Erratum for: DNA barcoding for industrial quality assurance. Planta Med. 83, 1117-1129. doi:10.1055/s-0043-113448

Shanmughanandhan, D., Ragupathy, S., Newmaster, S. G., Mohanasundaram, S., and Sathishkumar, R. (2016). Estimating herbal product authentication and adulteration in India using a vouchered, DNA-based biological reference material library. Drug Saf. 39, 1211-1227. doi:10.1007/s40264-016-0459-0

Shim, Y. H., Park, C. D., Kim, D. H., Cho, J. H., Cho, M. H., and Kim, H. J. (2005). Identification of Panax species in the herbal medicine preparations using gradient PCR method. Biol. Pharm. Bull. 28, 671-676. doi:10.1248/bpb.28.671

Simmler, C., Graham, J. G., Chen, S. N., and Pauli, G. F. (2018). Integrated analytical assets aid botanical authenticity and adulteration management. Fitoterapia 129, 401-414. doi:10.1016/j.fitote.2017.11.017
Tian, X., Lv, S., Tian, H., Wang, R., and Wang, H. (2020). Development of an accurate and reliable DNA method for botanical origin authentication of ginseng food products. J. Food Compos. Anal. 87, 103419. doi:10.1016/j.jfca. 2020.103419

Wallace, L. J., Boilard, S. M. A. L., Eagle, S. H. C., Spall, J. L., Shokralla, S., and Hajibabaei, M. (2012). DNA barcodes for everyday life: routine authentication of Natural Health Products. Food Res. Int. 49, 446-452. doi:10.1016/j.foodres.2012.07.048

Wang, K., Zeng, H., Zhang, Y., Xie, X., Yue, Z., Zhang, W., et al. (2019). A hierarchical screening method for detection of illegal adulterants in Fur seal ginseng pills by profiling analysis of HPLC multi-dimensional fingerprints. J. Sep. Sci. 42, 1509-1519. doi:10.1002/jssc.201900003

Wu, C.-S., Chen, Y.-H., Chen, C.-L., Chien, S.-K., Syifa, N., Hung, Y.-C., et al. (2019). Constructing a bilingual website with validated database for Herb and Western medicine interactions using Ginseng, Ginkgo and Dong Quai as examples. BMC Complement. Altern. Med. 19, 335. doi:10.1186/s12906-0192731-1

Xie, P., Chen, S., Liang, Y. Z., Wang, X., Tian, R., and Upton, R. (2006). Chromatographic fingerprint analysis--a rational approach for quality assessment of traditional Chinese herbal medicine. J. Chromatogr. A. 1112, 171-180. doi:10.1016/j.chroma.2005.12.091

Xie, X., Zhang, Y., Yue, Z., Wang, K., Mai, X., Liu, Y., et al. (2019). Multi-fingerprint profiling analysis for screening and quantification of illegal adulterated antidiabetics in a functional food using HPLC coupled to diode array detection/fluorescence detection. Microchemical Journal. 149, 103995. doi:10. 1016/j.microc.2019.103995

Yang, H.-Q., Dong, Y.-R., Gu, Z.-J., Liang, N., and Yang, J.-B. (2012). A preliminary assessment of mat $\mathrm{K}, \mathrm{rbc} \mathrm{L}$ and trn $\mathrm{H}-$ psb A as DNA barcodes for Calamus (Arecaceae) species in China with a note on ITS. Ann. Bot. Fenn. 49, 319-330. doi:10.5735/085.049.0603

Yang, M. S., and Wu, M. Y. (2016). Chinese ginseng. Nutraceuticals. 50, 693-705. doi:10.1016/B978-0-12-802147-7.00050-4

Yang, W., Qiao, X., Li, K., Fan, J., Bo, T., Guo, D. A., et al. (2016). Identification and differentiation of Panax ginseng, Panax quinquefolium, and Panax notoginseng by monitoring multiple diagnostic chemical markers. Acta Pharm. Sin. B. 6, 568-575. doi:10.1016/j.apsb.2016.05.005

Yao, C., Yang, W., Si, W., Pan, H., Qiu, S., Wu, J., et al. (2016). A strategy for establishment of practical identification methods for Chinese patent medicine from systematic multi-component characterization to selective ion monitoring of chemical markers: shuxiong tablet as a case study. RSC Adv. 6, 65055-65066. doi:10.1039/C6RA10883K

Yu, C., Wang, C. Z., Zhou, C. J., Wang, B., Han, L., Zhang, C. F., et al. (2014). Adulteration and cultivation region identification of American ginseng using HPLC coupled with multivariate analysis. J. Pharm. Biomed. Anal. 99, 8-15. doi:10.1016/j.jpba.2014.06.031

Yuk, J., Patel, D. N., Isaac, G., Smith, K., Wrona, M., Olivos, H. J., et al. (2016). Chemical profiling of ginseng species and ginseng herbal products using UPLC/ QTOF-MS. J. Braz. Chem. Soc. 27, 1476-1483. doi:10.5935/0103-5053.20160189

Zhang, P., Liu, C., Zheng, X., Wu, L., Liu, Z., Liao, B., et al. (2019). Full-length multi-barcoding: DNA barcoding from single ingredient to complex mixtures. Genes 10, 343. doi:10.3390/genes10050343

Zhao, N., Cheng, M., Lv, W., Wu, Y., Liu, D., and Zhang, X. (2020). Peptides as potential biomarkers for authentication of mountain-cultivated ginseng and cultivated ginseng of different ages using UPLC-HRMS. J. Agric. Food Chem. 68, 2263-2275. doi:10.1021/acs.jafc.9b05568

Zhou, Y. Q., Li, M., Wang, X. S., Xia, W., and Zhang, L. H. (2016). Development and evaluation of DNA detection kit for Panax ginseng C. A. Mey. Chinese Pharmaceutical Journal. 51, 1866-1870. 10.1109/BMEI.2014.7002854

Conflict of Interest: The authors declare that the research was conducted in the absence of any commercial or financial relationships that could be construed as a potential conflict of interest.

Copyright (c) 2021 Ichim and de Boer. This is an open-access article distributed under the terms of the Creative Commons Attribution License (CC BY). The use, distribution or reproduction in other forums is permitted, provided the original author(s) and the copyright owner(s) are credited and that the original publication in this journal is cited, in accordance with accepted academic practice. No use, distribution or reproduction is permitted which does not comply with these terms. 